\title{
Minimal Solutions of Three-Term Recurrence Relations and Orthogonal Polynomials*
}

\author{
By Walter Gautschi
}

\begin{abstract}
We observe that the well-known recurrence relation $p_{n+1}(z)=\left(z-a_{n}\right) p_{n}(z)-$ $b_{n} p_{n-1}(z)$ for orthogonal polynomials admits a "minimal solution" if $z$ is outside the spectrum of the mass distribution $d s(t)$ with respect to which the polynomials are orthogonal and if the moment problem for this distribution is determined. The minimal solution, indeed, is $f_{n}(z)=\int p_{n}(t) d s(t) /(z-t)$, and can be computed accurately by means of the author's continued fraction algorithm. An application is made to special Gauss-type quadrature formulas.
\end{abstract}

1. Introduction. Minimal solutions of three-term recurrence relations, and their computational implications, are discussed systematically in [7]. Effective algorithms for computing minimal solutions have been developed in [7], [16], [17] and continue to be the subject of further study (see, e.g., [14], [26], [5], [2], [24], [25], [3]). With these powerful algorithms at hand, it seems desirable to delineate large classes of recurrence relations for which the presence of minimal solutions can be ascertained and the minimal solution itself identified. Even more desirable is an understanding of the deeper reasons for the occurrence of minimal solutions, in terms of intrinsic features of the subject area in which they arise.

Few attempts have been made along these lines. The work of Thacher [22], [23] on power series solution of linear differential equations with polynomial coefficients may be considered a beginning, inasmuch as the presence of minimal solutions (of the recurrence relation satisfied by the expansion coefficients) is conjectured to be related in a specified way to the type of singularities in the differential equation. Here we wish to consider the recurrence relation

$$
y_{n+1}=\left(z-a_{n}\right) y_{n}-b_{n} y_{n-1}, \quad n=1,2,3, \ldots, b_{n}>0,
$$

associated with the moment sequence of a mass distribution $d s(t)$ on the real line. We make the simple observation, apparently overlooked so far, that for any $z$ outside the spectrum of $d s(t)$ the recurrence relation (1.1) possesses a (readily identifiable) minimal solution whenever the moment problem for $d s(t)$ is determined (Section 3). If the spectrum of $d s(t)$ is bounded (i.e., there is zero mass outside some finite interval), then the moment problem is always determined; hence a minimal solution always exists.

\footnotetext{
Received July 7, 1980.

1980 Mathematics Subject Classification. Primary 33A65, 39A10; Secondary 65D20, 65D30.

Key words and phrases. Minimal solutions of three-term recurrence telations, orthogonal polynomials, moment problem, modified moments.

*Sponsored in part by the National Science Foundation under grant MCS-7927158.
} 
Our observation opens up a large class of recurrence relations to which the algorithms mentioned above can be usefully applied. One such application (to special Gaussian quadrature rules) will be discussed in Section 4, and an appropriate algorithm in Section 5.

In the case of the Jacobi distribution on $[-1,1]$, the existence of a minimal solution (when $z \notin[-1,1]$ ) can easily be inferred from the asymptotic theory of difference equations [7, Theorem 2.3]. This is no longer the case for distributions on infinite intervals, such as the generalized Laguerre, or Hermite, distribution.

2. Orthogonal Polynomials and Associated Polynomials. Let $d s(t)$ be a mass distribution on the finite or infinite interval $[a, b]$ (i.e., $s(t)$ a nondecreasing function with infinitely many points of increase), and let $d s(t)$ admit finite moments of all orders,

$$
m_{n}=\int_{a}^{b} t^{n} d s(t), \quad n=0,1,2, \ldots
$$

Let $\left\{p_{n}(z)\right\}$ be the (monic) polynomials orthogonal on $[a, b]$ with respect to $d s(t)$,

$$
\int_{a}^{b} p_{n}(t) p_{m}(t) d s(t)=0, \quad n \neq m
$$

As is well known, they satisfy a three-term recurrence relation of the form

$$
\left\{\begin{array}{l}
p_{n+1}(z)=\left(z-a_{n}\right) p_{n}(z)-b_{n} p_{n-1}(z), \quad n=0,1,2, \ldots, \\
p_{-1}(z)=0, \quad p_{0}(z)=1,
\end{array}\right.
$$

where $a_{n}$ are real, and $b_{n}>0$, all $n$. ( $b_{0}$ is arbitrary, but will be set equal to $m_{0}$.)

The polynomials

$$
q_{n}(z)=\int_{a}^{b} \frac{p_{n}(z)-p_{n}(t)}{z-t} d s(t), \quad n=0,1,2, \ldots,
$$

are called the polynomials associated with the orthogonal polynomials $\boldsymbol{p}_{\boldsymbol{n}}$. It is easily seen (and well known) that they also satisfy the recurrence relation (2.3), at least for $n>1$. Indeed, by (2.3),

$$
\begin{aligned}
p_{n+1}(z) & -p_{n+1}(t) \\
= & z p_{n}(z)-t p_{n}(t)-a_{n}\left[p_{n}(z)-p_{n}(t)\right]-b_{n}\left[p_{n-1}(z)-p_{n-1}(t)\right] \\
& =(z-t) p_{n}(t)+\left(z-a_{n}\right)\left[p_{n}(z)-p_{n}(t)\right]-b_{n}\left[p_{n-1}(z)-p_{n-1}(t)\right],
\end{aligned}
$$

hence, dividing by $z-t$ and integrating,

$$
\left\{\begin{array}{l}
q_{n+1}(z)=\int_{a}^{b} p_{n}(t) d s(t)+\left(z-a_{n}\right) q_{n}(z)-b_{n} q_{n-1}(z), \quad n=0,1,2, \ldots \\
q_{-1}(z)=0, \quad q_{0}(z)=0
\end{array}\right.
$$

By orthogonality, the integral on the right of (2.5) vanishes if $n>0$, and equals $m_{0}$, if $n=0$. Consequently,

$$
\left\{\begin{array}{l}
q_{n+1}(z)=\left(z-a_{n}\right) q_{n}(z)-b_{n} q_{n-1}(z), \quad n=1,2,3, \ldots, \\
q_{0}(z)=0, \quad q_{1}(z)=m_{0} .
\end{array}\right.
$$


((2.6) also holds for $n=0$, if we redefine $q_{-1}(z)=-1$ and assume $b_{0}=m_{0}$.) Since $p_{0}(z)=1, p_{1}(z)=z-a_{0}$, hence the Wronskian of $p_{n}, q_{n}$ is equal to $m_{0}$ at $n=0$, we see that $\left\{p_{n}(z)\right\}$ and $\left\{q_{n}(z)\right\}$ are two linearly independent solutions of the recurrence relation (1.1).

3. Minimal Solutions and the Moment Problem. A solution $f_{n}$ of the recurrence relation (1.1) is said to be minimal [7] if there exists a linearly independent solution, $g_{n}$, of the same recurrence relation such that

$$
\lim _{n \rightarrow \infty} \frac{f_{n}}{g_{n}}=0 .
$$

We show that the existence of a minimal solution is closely related to the determinacy of the moment problem for $d s(t)$.

Let

$$
F(z)=\int_{a}^{b} \frac{d s(t)}{z-t}, \quad z \notin[a, b] .
$$

It is known [18, Satz 4.1] that the integral (3.2) has an "associated continued fraction"

$$
F(z) \sim \frac{b_{0}}{z-a_{0}-} \frac{b_{1}}{z-a_{1}-} \frac{b_{2}}{z-a_{2}-} \cdots\left(b_{0}=m_{0}\right),
$$

where $a_{n}, b_{n}$ are the same coefficients as those appearing in the recurrence relation (1.1). Furthermore, the $n$th convergent of the continued fraction in (3.3) is equal to $q_{n} / p_{n}$, where $p_{n}, q_{n}$ are defined in (2.3) and (2.6), respectively,

$$
\frac{b_{0}}{z-a_{0}-} \frac{b_{1}}{z-a_{1}-} \cdots \frac{b_{n-1}}{z-a_{n-1}}=\frac{q_{n}(z)}{p_{n}(z)}, \quad n=1,2,3, \ldots
$$

We are interested in the case in which the continued fraction in (3.3) converges to the integral $F(z)$,

$$
\lim _{n \rightarrow \infty} \frac{q_{n}(z)}{p_{n}(z)}=F(z), \quad z \notin[a, b] .
$$

If (3.5) holds true, then indeed

$$
f_{n}(z)=F(z) p_{n}(z)-q_{n}(z)
$$

is a minimal solution of (1.1). This follows at once by observing that (3.5) implies

$$
\frac{f_{n}(z)}{p_{n}(z)}=F(z)-\frac{q_{n}(z)}{p_{n}(z)} \rightarrow 0 \quad \text { as } n \rightarrow \infty .
$$

In view of (2.4) and (3.2), we can write $f_{n}$ in the alternative form

$$
f_{n}(z)=\int_{a}^{b} \frac{p_{n}(t) d s(t)}{z-t}, \quad z \notin[a, b], \quad n=0,1,2, \ldots
$$

If we define (see the parenthetic remark after (2.6)) $b_{0}=m_{0}, q_{-1}(z)=-1$, then $f_{n}$ in (3.6) satisfies (1.1) not only for $n>1$, but also for $n=0$, and we obtain the convenient starting value

$$
f_{-1}(z)=1
$$


Any condition which guarantees (3.5) is a sufficient condition for (3.8) to be a minimal solution of (1.1). We now recall some such conditions. If the interval $[a, b]$ is finite, then (3.5) always holds, by virtue of Markov's theorem [18, Satz 4.2]. If $[a, b]$ is a half-infinite interval, say $[0, \infty]$, we have (3.5) if the Stieltjes moment problem for the moment sequence (2.1) is determined [18, Satz 4.14 and Satz 4.10]. A sufficient condition for the latter, due to Carleman, is [20, Theorem 1.11]

$$
\sum_{n=1}^{\infty} m_{n}^{-1 / 2 n}=\infty
$$

Similarly, for the doubly-infinite interval $[-\infty, \infty]$ (in which case $z$ in (3.5) is necessarily complex), the validity of (3.5) is assured if the Hamburger moment problem for the moment sequence (2.1) is determined [18, Satz 4.15 and Satz 4.11]. Sufficient conditions for the latter, due to Carleman, are [20, Theorem 1.10 and p. 59]

$$
\sum_{n=1}^{\infty} m_{2 n}^{-1 / 2 n}=\infty, \text { or } \sum_{n=1}^{\infty} b_{n}^{-1 / 2}=\infty
$$

Since, for a finite interval $[a, b]$, the moment problem for (2.1) is always determined [20, Corollary 1.1], we may summarize by saying that condition (3.5) is satisfied, hence $f_{n}(z)$ in (3.8) is a minimal solution of (1.1), whenever the moment problem for the moment sequence (2.1) is determined. Most distributions $d s(t)$ that arise in practice indeed correspond to a determined moment problem.

4. Generation of Special Gaussian Quadrature Rules. Let $d s(t)$ be a mass distribution, as in Section 2, and assume first that its support is a (finite or infinite) subinterval $[a, b]$ of the real line. Let $x$ be real, outside of $[a, b]$, and consider the new distribution

$$
d \sigma(t)=\frac{d s(t)}{|x-t|}, \quad a<t<b, \quad x \in \mathbf{R} \backslash[a, b] .
$$

Given the recurrence relation (2.3) for the orthogonal polynomials $\left\{p_{n}\right\}$ associated with $d s(t)$ we are interested in constructing the (monic) orthogonal polynomials $\left\{\pi_{n}\right\}$ with respect to $d \sigma(t)$, and the corresponding Gaussian quadrature rules, all of which clearly exists uniquely.

A good general procedure for accomplishing our task consists in first determining the coefficients $\alpha_{n}, \beta_{n}$ for the desired polynomials,

$$
\left\{\begin{array}{l}
\pi_{n+1}(z)=\left(z-\alpha_{n}\right) \pi_{n}(z)-\beta_{n} \pi_{n-1}(z), \quad n=0,1,2, \ldots, \\
\pi_{-1}(z)=0, \quad \pi_{0}(z)=1,
\end{array}\right.
$$

in terms of the coefficients $a_{n}, b_{n}$ of the given orthogonal polynomials and in terms of the "modified moments" of $d \sigma(t)$,

$$
\nu_{n}=\int_{a}^{b} \frac{p_{n}(t) d s(t)}{|x-t|}, \quad n=0,1,2, \ldots,
$$

and then to compute the eigenvalues and first components of the corresponding eigenvectors of the tridiagonal symmetric Jacobi matrix (with elements $\alpha_{n}, n=$ $0,1,2, \ldots$, on the main diagonal, and elements $\sqrt{\beta_{n}}, n=1,2,3, \ldots$, on the side diagonals), using the implicit $Q L$ algorithm. For details see [8, Section 5], [10], 
[9]. Although this approach may not be the best possible, in terms of efficiency (for more direct methods, see [11], [19]), it has the distinct advantage of numerical stability, particularly when the interval $[a, b]$ is finite. Essential for the success of this approach, however, is the accurate determination of the modified moments in (4.3). These are seen to be identical with $f_{n}(x)$ (or $-f_{n}(x)$, if $x<a$ ) in (3.8)-a minimal solution of the recurrence relation (1.1), if the moment problem for $d s(t)$ is determined. An effective algorithm for the computation of this minimal solution will be discussed in Section 5 .

A similar application can be made to weight distributions of the type (cf. also [12])

$$
d \sigma(t)=\frac{d s(t)}{(x-t)^{2}+y^{2}}, \quad a \leqslant t \leqslant b, \quad x \in \mathbf{R}, \quad y>0,
$$

where the support $[a, b]$ of $d s(t)$ may or may not coincide with the whole real line. The modified moments, in this case, are given by

$$
\nu_{n}=\int_{a}^{b} \frac{p_{n}(t) d s(t)}{(x-t)^{2}+y^{2}}, \quad n=0,1,2, \ldots
$$

They are easily expressed in terms of $f_{n}(z)$ in (3.8). Indeed, letting $z=x+i y$, and observing that

$$
\frac{1}{(x-t)^{2}+y^{2}}=-\frac{1}{2 i y}\left(\frac{1}{z-t}-\frac{1}{\bar{z}-t}\right),
$$

one finds immediately

$$
\nu_{n}=-\frac{\operatorname{Im} f_{n}(z)}{\operatorname{Im} z}
$$

Thus again, in the case of determinacy, we can generate $\nu_{n}$ accurately in terms of the minimal solution $f_{n}(z)$ of (1.1).

5. An Algorithm for Calculating the Minimal Solution $f_{n}(z)$. A number of algorithms are known for computing minimal solutions of a three-term recurrence relation: Miller's backward recurrence algorithm [1, p. xvii], the author's algorithm based on continued fractions [7], Olver's algorithm [16], and an economic reformulation of Olver's algorithm due to Van der Cruyssen [24]. Our experience with these algorithms, when applied to some typical minimal solutions $f_{n}(z)$, has been mixed. All algorithms, but the author's, have proved prone to overflow, particularly when $x$ is moderately close to the half-infinite interval $[a, b]=[0, \infty]$ in $(4.1)$, and $z=x+i y$ moderately close to $[a, b]=[-\infty, \infty]$ in (4.4). The algorithms converge very slowly in these cases. We have tried to combat overflow in Olver's algorithm by rewriting it in terms of appropriate ratios, but were not entirely successful. (An alternative way of dealing with the overflow problem, at the expense of approximately doubling the work, is to use extended-range arithmetic packages, as in [21], [13].) On the other hand, our own algorithm in [7], although not subject to overflow conditions, requires good estimates of the starting index (for backward recurrence) to remain competitive with the other algorithms. Fortunately, such estimates can be derived for the most common distributions $d s(t)$ occurring in practice. For this reason, in the present context, we tend to prefer the continued fraction algorithm of 
[7] over the other algorithms mentioned. As "normalizing condition" we use the simple condition (3.9), which obviates the need of computing $f_{0}(z)$ separately.

Suppose, then, that we wish to compute $f_{n}(z)$ for $n=0,1,2, \ldots, N$. Let $\nu>N$, and let quantities $r_{n}^{(\nu)}$ and $f_{n}^{(v)}$ be generated according to

$$
\begin{cases}r_{\nu}^{(\nu)}=0, & r_{n-1}^{(\nu)}=\frac{b_{n}}{z-a_{n}-r_{n}^{(\nu)}}, \quad n=\nu, \nu-1, \ldots, 1,0, \\ f_{-1}^{(v)}=1, & f_{n}^{(\nu)}=r_{n-1}^{(\nu)} f_{n-1}^{(\nu)}, \quad n=0,1,2, \ldots, N .\end{cases}
$$

(Recall that $b_{0}=m_{0}$.) If $f_{n}(z)$ is a minimal solution of $(1.1)$, then [7, p. 39]

$$
\lim _{\nu \rightarrow \infty} f_{n}^{(\nu)}=f_{n}(z), \quad n=0,1,2, \ldots, N .
$$

To implement (5.1), (5.2), one starts with some initial value of the index $\nu$ and keeps increasing $\nu$, say by 5 , until $\left|f_{n}^{(\nu+5)}-f_{n}^{(v)}\right|<\varepsilon\left|f_{n}^{(\nu+5)}\right|$ for all $n=$ $0,1,2, \ldots, N$, where $\varepsilon$ is an appropriate (relative) error tolerance. (Alternatively, one could apply the convergence test on the $r_{n-1}^{(v)}$, rather than the $f_{n}^{(v)}$, and thus avoid the computation of $f_{n}^{(v)}$ for all but the final value of $\nu$.)

For this algorithm to be effective, it is imperative that realistic estimates be available for the initial value of the index $\nu$, given $N$ and the error tolerance $\varepsilon$. Such estimates can be derived from known asymptotic results [6] concerning the behavior of $f_{n}(z)$ and $p_{n}(z)$ for large $n$.

To begin with, we recall from $[7,(3.18)]$ that the relative errors can be approximated by

$$
\varepsilon_{n}^{(v)} \div \frac{f_{\nu+1}}{g_{\nu+1}}\left(g_{-1}-\frac{g_{n}}{f_{n}}\right), \quad n=0,1,2, \ldots, N
$$

where $\left\{g_{n}\right\}$ is any solution of (1.1) which is linearly independent of the (minimal) solution $\left\{f_{n}\right\}$. In view of (3.7), we may choose $g_{n}=p_{n}(z)$, in which case $g_{-1}=0$. Since $\left|p_{n} / f_{n}\right| \rightarrow \infty$ as $n \rightarrow \infty$, we have, for $N$ sufficiently large,

$$
\max _{1<n<N}\left|\varepsilon_{n}^{(v)}\right| \doteqdot\left|\varepsilon_{N}^{(v)}\right| \doteqdot\left|\frac{f_{v+1}}{p_{\nu+1}} / \frac{f_{N}}{p_{N}}\right| .
$$

The ratios $f_{\nu+1} / p_{\nu+1}(\nu>N)$ and $f_{N} / p_{N}$ in (5.4) can be estimated, at least for some common weight distributions $d s(t)$, from the asymptotic formulas for $f_{n} / p_{n}$ given in the Appendix of [6].

For the Jacobi distribution $d s(t)=(1-t)^{\alpha}(1+t)^{\beta} d t$ on $[-1,1]$, where $\alpha>-1$, $\beta>-1$, for example, using Eq. (A.1) in [6], one finds for $z$ bounded away from $[-1,1]$ that

$$
\frac{f_{\nu+1}(z)}{p_{v+1}(z)} / \frac{f_{N}(z)}{p_{N}(z)} \sim\left\{\frac{1}{z+(z-1)^{1 / 2}(z+1)^{1 / 2}}\right\}^{2(v+1-N)}, \quad \nu>N \rightarrow \infty,
$$

independently of $\alpha$ and $\beta$. (In evaluating the square roots in (5.5), the principal values of $\arg (z-1)$ and $\arg (z+1)$ are to be used.) For the maximum error in (5.4) to be less than $\varepsilon$, we thus find for $\nu$ the estimate

$$
\nu>N+\frac{\ln (1 / \varepsilon)}{2 \ln \left|z+(z-1)^{1 / 2}(z+1)^{1 / 2}\right|} .
$$


This is applicable to modified moments of the type (4.5), with Jacobi distribution $d s(t)$, in which case $z=x+i y$ in (4.6) is complex, with $y>0$. The estimate (5.6) also applies to (4.3), where $z=x$ is real outside of $[-1,1]$, and can then be written in the simpler form

$$
\nu \geqslant N+\frac{\ln (1 / \varepsilon)}{2 \ln \left(|x|+\sqrt{x^{2}-1}\right)}, \quad x \in \mathbf{R} \backslash[-1,1]
$$

The contours in (5.6), i.e., the lines of constant $\left|z+\sqrt{z^{2}-1}\right|=\rho>1$, are ellipses with foci at \pm 1 and sum of semiaxes equal to $\rho$; cf. [4, p. 19].

In the case of generalized Laguerre distributions $d s(t)=t^{\alpha} e^{-t} d t$ on $[0, \infty]$, where $\alpha>0$, we apply Eq. (A.6) in [6] and combine this with the well-known asymptotic formulas [15, 9.7.1 and 9.7.2] for modified Bessel functions, to obtain,

$$
\begin{aligned}
& \frac{f_{\nu+1}(z)}{p_{\nu+1}(z)} / \frac{f_{N}(z)}{p_{N}(z)} \\
& \sim \exp \left\{-4\left[\sqrt{\nu+1-\frac{\alpha+1}{2}}-\sqrt{N+\frac{\alpha+1}{2}}\right]\left(z e^{-i \pi}\right)^{1 / 2}\right\}, \\
& \nu>N \rightarrow \infty .
\end{aligned}
$$

Here, $z$ is assumed fixed in the complex plane cut along the positive real axis, and $0<\arg z<2 \pi$. For our maximum error in (5.4) to be less than $\varepsilon$, it suffices to choose $\nu$ such that

$$
\begin{array}{r}
\nu>\left[\sqrt{N+\frac{\alpha+1}{2}}+\frac{\ln (1 / \varepsilon)}{4 \sqrt{|z|} \cos \frac{1}{2}(\varphi-\pi)}\right)^{2}-\frac{\alpha+1}{2}, \\
0<\varphi=\arg z<2 \pi .
\end{array}
$$

The contours in (5.8), i.e., the lines of constant $\sqrt{|z|} \cos \frac{1}{2}(\varphi-\pi)=\sqrt{\rho}$, are now parabolas with focus at the origin and vertex at $(-\rho, 0)$.

Finally, the case of Hermite distribution $d s(t)=e^{-t^{2}} d t$ on $(-\infty, \infty)$ can be reduced to the case $\alpha= \pm \frac{1}{2}$ of the generalized Laguerre distribution by observing that $H_{2 n}(z)=L_{n}^{(-1 / 2)}\left(z^{2}\right), H_{2 n+1}(z)=z L_{n}^{(1 / 2)}\left(z^{2}\right)$, hence, for $z$ nonreal,

$$
\int_{-\infty}^{\infty} \frac{e^{-t^{2}} H_{n}(t) d t}{z-t}=\delta_{n}(z) \int_{0}^{\infty} \frac{e^{-t} t^{ \pm 1 / 2} L_{[n / 2]}^{( \pm 1 / 2)}(t) d t}{z^{2}-t},
$$

where + or - holds, depending on whether $n$ is odd or even, and where $\delta_{n}(z)=1$ for $n$ odd, and $\delta_{n}(z)=z$ for $n$ even. Proceeding as before, we now find for $\nu$ the estimate

$$
\nu>2\left(\sqrt{\frac{N}{2}}+\frac{\ln (1 / \varepsilon)}{4|z| \sin \varphi}\right)^{2}, \quad 0<\varphi=\arg z<\pi .
$$

The contours in (5.9) are straight lines parallel to the real axis.

Numerical experience has shown the estimates (5.6), (5.8), (5.9) to be quite realistic. In the majority of cases examined, one repetition of the algorithm (5.1) (with $\nu$ incremented by 5) suffices to confirm the desired accuracy. Occasionally, 
two repetitions are required, and only rarely three (and this only when $\nu$ is quite large). On the other hand, when lowered by 5, the estimates, with few exceptions, proved inadequate to achieve the desired accuracy.

Department of Computer Sciences

Purdue University

West Lafayette, Indiana 47907

1. British Association for the Advancement of ScIEnce, Mathematical Tables, vol. X, Bessel Functions, Part II, Functions of Positive Integer Order, Cambridge Univ. Press, Cambridge, 1952.

2. J. R. CASH, "An extension of Olver's method for the numerical solution of linear recurrence relations," Math. Comp., v. 32, 1978, pp. 497-510.

3. J. R. CASH, "A note on Olver's algorithm for the solution of second-order linear difference equations," Math. Comp., v. 35, 1980, pp. 767-772.

4. P. J. DAVIS, Interpolation and Approximation, Blaisdell, New York, 1963.

5. P. Deuflhard, "A summation technique for minimal solutions of linear homogeneous difference equations," Computing, v. 18, 1977, pp. 1-13.

6. J. D. Donaldson \& D. Elliotr, "A unified approach to quadrature rules with asymptotic estimates of their remainders," SIAM J. Numer. Anal., v. 9, 1972, pp. 573-602.

7. W. GAUTSCHI, "Computational aspects of three-term recurrence relations," SIAM Rev., v. 9, 1967, pp. 24-82.

8. W. GAutschI, "Questions of numerical condition related to polynomials," in Recent Advances in Numerical Analysis (C. de Boor and G. H. Golub, Eds.), Academic Press, New York, 1978, pp. 45-72.

9. W. GAUTSCHI, "On generating Gaussian quadrature rules," in Numerische Integration, ISNM Vol. 45 (G. Hämmerlin, Ed.), Birkhäuser Verlag, Basel, 1979, pp. 147-154.

10. G. H. GoluB \& J. H. WelsCh, "Calculation of Gauss quadrature rules," Math. Comp., v. 23, 1969, pp. 221-230.

11. R. Kumar, “A class of quadrature formulas," Math. Comp., v. 28, 1974, pp. 769-778.

12. R. Kumar, "Certain Gaussian quadratures," J. Inst. Math. Appl., v. 14, 1974, pp. 175-182.

13. D. W. LozIER \& J. M. SMTrH, "Algorithm 567-Extended-range arithmetic and normalized Legendre polynomials," ACM Trans. Math. Software, v. 7, 1981. (To appear.)

14. R. M. M. MATtheU \& A. vAN DER Sluis, "Error estimates for Miller's algorithm," Numer. Math., v. 26, 1976, pp. 61-78.

15. F. W. J. Olver, "Bessel functions of integer order," Handbook of Mathematical Functions with Formulas, Graphs, and Mathematical Tables (M. Abramowitz and I. A. Stegun, Eds.), Nat. Bur. Standards, Appl. Math. Ser., no. 55, Superintendent of Documents, U.S. Government Printing Office, Washington, D.C., 1964, pp. 355-433.

16. F. W. J. OLVER, "Numerical solution of second-order linear difference equations," J. Res. Nat. Bur. Standards, v. 71B, 1967, pp. 111-129.

17. F. W. J. Olver \& D. J. SOOKNE, "Note on backward recurrence algorithms," Math. Comp., v. 26, 1972, pp. 941-947.

18. O. Perron, Die Lehre von den Kettenbrüchen, Vol. II, Teubner Verlag, Stuttgart, 1957.

19. T. E. PRICE, JR., "Orthogonal polynomials for nonclassical weight functions," SIAM J. Numer. Anal., v. 16, 1979, pp. 999-1006.

20. J. A. Shoнat \& J. D. Tamarkin, The Problem of Moments, Math. Surveys, No. I, Amer. Math. Soc., Providence, R.I., 1943.

21. J. M. Smith, F. W. J. Olver \& D. W. Lozier, "Extended-range arithmetic and normalized Legendre polynomials," ACM Trans. Math. Software, v. 7, 1981.(To appear.)

22. H. C. ThACHER, JR., "Series solutions to differential equations by backward recurrence," Proc. IFIP Congress 71, Vol. 2, North-Holland, Amsterdam, 1972, pp. 1287-1291.

23. H. C. ThACHER, JR., "New backward recurrences for Bessel functions," Math. Comp., v. 33, 1979, pp. 744-764.

24. P. van Der CRUYSSEN, "A reformulation of Olver's algorithm for the numerical solution of second-order linear difference equations," Numer. Math., v. 32, 1979, pp. 159-166.

25. P. VAN DER CRUYSSEN, "Linear difference equations and generalized continued fractions," Computing, v. 22, 1979, pp. 269-278.

26. R. V. M. ZAHAR, "A mathematical analysis of Miller's algorithm," Numer. Math., v. 27, 1977, pp. 427-447. 